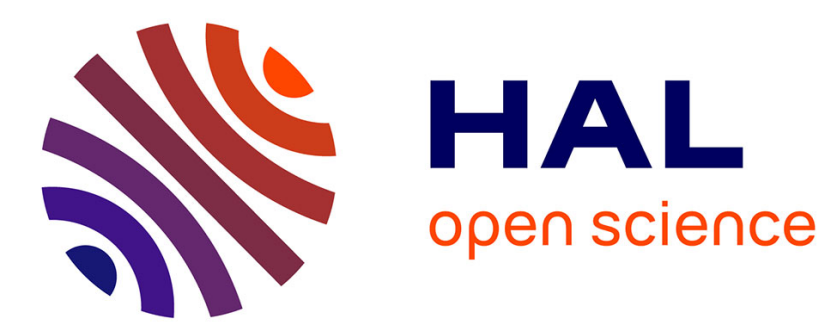

\title{
Religion et modernité dans les récits des élèves
}

Sébastien Urbanski

\section{To cite this version:}

Sébastien Urbanski. Religion et modernité dans les récits des élèves. Françoise Lantheaume; Jocelyn Létourneau. Le récit du commun : l'histoire nationale racontée par les élèves, Presses Universitaires de Lyon; Presses universitaires de Lyon, pp.101-116, 2016, 978-2-7297-0907-5. 10.4000/books.pul.23779 . halshs-01494199

\section{HAL Id: halshs-01494199 \\ https://shs.hal.science/halshs-01494199}

Submitted on 22 Apr 2020

HAL is a multi-disciplinary open access archive for the deposit and dissemination of scientific research documents, whether they are published or not. The documents may come from teaching and research institutions in France or abroad, or from public or private research centers.
L'archive ouverte pluridisciplinaire HAL, est destinée au dépôt et à la diffusion de documents scientifiques de niveau recherche, publiés ou non, émanant des établissements d'enseignement et de recherche français ou étrangers, des laboratoires publics ou privés. 


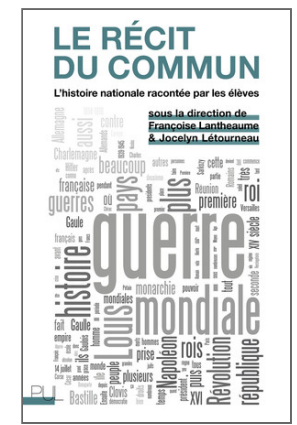

Françoise Lantheaume et Jocelyn Létourneau (dir.)

Le récit du commun

L'histoire nationale racontée par les élèves

Presses universitaires de Lyon

\section{Religion et modernité dans les récits des élèves}

\section{Sébastien Urbanski}

DOl : 10.4000/books.pul.23779

Éditeur : Presses universitaires de Lyon

Lieu d'édition : Lyon

Année d'édition : 2016

Date de mise en ligne : 29 novembre 2019

Collection : Histoire

ISBN électronique : 9782729711580

\section{Sbooks}

http://books.openedition.org

\section{Édition imprimée}

Date de publication : 1 janvier 2016

\section{Référence électronique}

URBANSKI, Sébastien. Religion et modernité dans les récits des élèves In : Le récit du commun : L'histoire nationale racontée par les élèves [en ligne]. Lyon : Presses universitaires de Lyon, 2016 (généré le 28 mars 2020). Disponible sur Internet : <http://books.openedition.org/pul/23779>. ISBN : 9782729711580. DOI : https://doi.org/10.4000/books.pul.23779. 


\section{RELIGION ET MODERNITÉ DANS LES RÉCITS DES ÉLĖVES}

SÉBASTIEN URBANSKI

L'analyse des références à la religion dans le corpus français nécessite dans un premier temps de situer la spécificité de la modernité «à la française " dans son rapport à la religion. À ce titre, il est utile de distinguer sécularisation et laïcisation. Le premier phénomène concerne d'abord les pays européens à majorité protestante où, en raison de la rupture avec le Vatican, le désenchantement du monde a touché conjointement les églises et les États. Le processus de laïcisation concerne quant à lui les pays à tradition catholique plus marquée, dans lesquels «l'émergence d'une sphère publique dégagée de l'emprise de l'Église romaine n'a pu passer que par une intervention volontariste, voire chirurgicale, du pouvoir politique " (Gauchet, 1998, p. 16). Ainsi, en France, pays de tradition catholique dans lequel l'État fut l'un des principaux opérateurs de «sortie de la religion ", une certaine méfiance subsiste à l'égard des religions, en tout cas dans leur dimension institutionnelle. Dans les débats contemporains plutôt vifs sur la laïcité scolaire, cette méfiance - qui est d'abord une construction politique - est perçue par certains comme étant difficilement conciliable avec les défis auxquels l'école doit faire face. Selon François Dubet, par exemple, l'hétérogénéité culturelle des élèves devrait nous conduire à "redéfinir les relations entre ce qui est vu comme un particularisme et ce qui est conçu comme universel »: dans ce cadre, il faudrait "renégocier la laïcité " dans la mesure où celle-ci, historiquement liée au christianisme et au colonialisme, participe d'un «particularisme national» (Dubet, 2012, p. 9). Au contraire, d'autres pensent que la généalogie laïque à la française, très cohérente et génératrice d'une saine allergie aux intégrismes, est particulièrement adaptée à notre temps (Kintzler, 2013). Quoi qu'il en soit, l'idée d'une posture spécifiquement française vis-à-vis des religions est largement partagée.

La présente enquête sur les récits est susceptible de fournir quelques éléments de réponse à la question de savoir ce qu'il en est chez les élèves. Ces derniers sont-ils imprégnés d'une méfiance à l'égard des religions ? Leur vision de l'histoire inclut-elle la dynamique décrite par Marcel Gauchet, qui veut que religion et politique soient marquées de lourds antagonismes ? Si tel est le cas, la religion est-elle perçue par les jeunes comme un phénomène essentiellement négatif ? Du coup, laïcisation et laïcité sont-elles envisagées par eux comme constitutives de l'histoire française? Par ailleurs, si la France peut être considérée comme un «idéaltype de la 
sécularité moderne, sur le terrain des institutions aussi bien que sur celui des consciences » (Hervieu-Léger, 2003, p. 292), les récits permettent-ils d'explorer l'articulation de ces deux dimensions ? Notre corpus donne en effet à voir des points de vue subjectifs, ceux des élèves, mais permet également de découvrir le travail institutionnel "objectif" susceptible d'influer sur leurs représentations. La laïcisation des institutions trouvet-elle écho dans l'histoire subjective racontée par les élèves? Si oui, quel est le rôle de l'enseignement scolaire dans cette médiation ?

Le corpus disponible, hors post-bac, permet d'éclairer ces questions. Si l'on regarde le tableau 1 , où sont regroupées les données sur lesquelles nous appuyons notre propos, la première chose qui frappe est la grande différence existant entre récits métropolitains et réunionnais. Si, dans leurs récits, les métropolitains insistent davantage sur la disparition progressive de l'emprise religieuse au cours de l'histoire, les Réunionnais expriment une vision de la modernité comme coexistence de différentes religions.

Par exemple, les seconds insistent davantage sur les religions comme phénomène social (voir infra, "Comparaison avec La Réunion »), tandis que les premiers pensent plutôt en termes d'emprise de "Dieu ", notamment via la monarchie absolue de droit divin et les guerres de religion, emprise finalement levée grâce à l'avènement de la république. Ainsi, Dieu est paradoxalement très présent dans l'histoire racontée par les métropolitains alors même que leurs représentations sont plus séculières que celles des Réunionnais.

Concentrons-nous d'abord sur les récits métropolitains, au nombre de $5086^{1}$. L'islam et le judaïsme sont beaucoup moins présents que le christianisme. La dimension culturelle et cultuelle de la religion est moins présente que sa dimension politique, avec notamment 71 occurrences du mot «clergé ». Les 28 occurrences du terme "pape » sont également très politisées, le pontife ne faisant quasiment rien d'autre que " couronner " les rois et « commander les croisades", que ce soit "pour reconquérir Jérusalem ou encore l'Espagne. » [récit no 440, lycée] La Bible est évoquée 15 fois ; dans la moitié des cas, c'est toutefois pour faire état de l'invention de l'imprimerie ("la Bible de Gutenberg»). Les baptêmes de rois, notamment celui de Clovis, sont mentionnés 334 fois, ce qui est considérable, mais leur signification religieuse est rarement développée. Dans la très grande majorité des cas, il s'agit surtout de couronnements - «les Francs sont arrivés, ont conquis la France et Clovis a été baptisé roi de France.» [récit $n^{\circ} 5645$, collège] - et l'événement tient souvent une fonction de repérage dans le temps : «Clovis est baptisé à Reims $\rightarrow$ début du moyen Age.» [récit no 4785, lycée] Le baptême de Clovis est en réalité perçu comme un événement politique bien plus que religieux, comme on le verra plus loin.

1. Le corpus "métropolitain " inclut les récits provenant de l'académie de Corse. La comparaison avec l'île de La Réunion est proposée plus loin dans ce chapitre (p. 112114). 
Tableau 1. Présence de la religion dans le corpus des récits métropolitains $(\mathrm{N}=5086)$ et réunionnais $(\mathrm{N}=737)$.

\begin{tabular}{|l|c|c|c|}
\hline \multirow{2}{*}{ Mots / termes et leurs dérivés } & \multicolumn{3}{|c|}{ Nombre d'occurrences } \\
\hline & $\begin{array}{c}\text { Récits } \\
\text { métropolitains }\end{array}$ & $\begin{array}{c}\text { Récits } \\
\text { réunionnais }\end{array}$ & Total \\
\hline Baptême & 334 & 7 & 341 \\
\hline Religion & 171 & 46 & 217 \\
\hline Laïcité & 64 & 3 & 67 \\
\hline Chrétien & 156 & 6 & 162 \\
\hline Protestant & 74 & 2 & 76 \\
\hline Clergé & 71 & 1 & 72 \\
\hline Catholique & 66 & 5 & 71 \\
\hline Jésus /le Christ ${ }^{\star}$ & 53 & 2 & 55 \\
\hline Pape & 28 & 0 & 28 \\
\hline Islam ${ }^{\star \star}$ & 15 & 12 & 27 \\
\hline Bible & 15 & 0 & 15 \\
\hline Judaïsme & 2 & 0 & 2 \\
\hline Dieu & 51 & 0 & 51 \\
\hline
\end{tabular}

* Les passages du type : «De 52 à 58 avant Jésus-Christ, la Gaule fut conquise par Jules César " [récit n ${ }^{\circ} 5705$, collège] n'ont pas été comptabilisés.

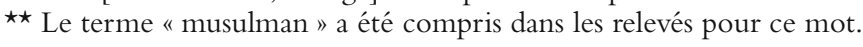

Ce bref survol du corpus métropolitain suggère que la religion est loin d'occuper une place centrale dans les représentations des élèves concernant l'histoire de France. Mais elle est assez présente pour les besoins d'une analyse approfondie, que l'on fera en deux temps. Dans la première partie, nous proposerons une analyse quantitative consistant à repérer des corrélations entre le nombre d'occurrences de tel ou tel terme (chrétien, pape, croisade...) et certaines variables (sexe, niveau scolaire, type d'établissement...). Dans la seconde partie, nous nous concentrerons moins sur les termes utilisés que sur le contenu même des récits : on distinguera des récits "anticléricaux ", "pro-chrétiens ", etc., qui seront mis en lien avec diverses variables à disposition. L'objectif est de dégager progressivement les lignes de forces et la dynamique globale du corpus recueilli en métropole.

\section{LA FRÉQUENCE DES RÉFÉRENCES À LA RELIGION DANS LE CORPUS MÉTROPOLITAIN}

La première donnée digne d'intérêt veut que les récits qui ont pour source principale des "cours déjà suivis durant la scolarité " insistent particulièrement sur l'aspect institutionnel et conflictuel des religions. Par 
exemple, la mobilisation des termes « croisade ", « clergé » ou « laïcité » est fortement corrélée avec la variable cours alors que des termes comme " religion", " dieu " ou "chrétien " ne le sont pas. L'enseignement scolaire n'est donc pas sans influence sur les représentations des élèves qui, comme on le verra, décrivent les religions en tant que phénomène avant tout politique. Par ailleurs, les garçons parlent 3 fois plus souvent des croisades et du pape que les filles, ce qui peut s'expliquer par leur vision différente de la guerre en général ${ }^{2}$, le pape étant souvent dépeint comme avide de conquêtes. En même temps, les croisades ne sont pas des guerres comme les autres ; dans la très grande majorité des cas, elles sont décrites comme ayant des causes religieuses : «Ce pays a une histoire pathétique : des guerres perdues et des croisades pour des dieux qui n'existent pas ", affirme un élève de lycée [récit $n^{\circ} 4176$ ] ; "Puissance religieuse $\rightarrow$ les croisades, discrimination en tout genre ", déclare un autre élève, de lycée également [récit no 4901]. On verra que, globalement, les élèves considèrent la religion comme un phénomène négatif. Par ailleurs, les garçons parlent bien plus souvent de "catholicisme » ou de "catholique(s) " que les filles. De même, ils mentionnent 2 fois plus souvent l'Église comme institution (en usant d'une majuscule pour orthographier le terme), et ce par contraste à "église " (écrit avec une minuscule), terme surtout privilégié par les filles et qui renvoie davantage à la dimension culturelle et patrimoniale. Ce dernier phénomène est sans doute lié à la politisation de la religion qu'on retrouve en particulier chez les garçons, des travaux en sociologie du genre ayant montré qu'en général, les garçons manifestent plus d'intérêt pour la politique et de façon plus précoce que les filles, qui développent souvent une attitude de retrait (Bargel, 2013). On peut aussi repérer des perceptions différentes entre garçons et filles sur des sujets historiques sensibles comme l'extermination des juifs d'Europe (ou ce qu'on appelle parfois " mémoire de la Shoah»), les premiers étant plus centrés sur les combats militaires, les batailles, les armes, et les secondes sur l'émotion, la compassion envers les victimes, mais aussi sur les histoires familiales. Peut-être ce phénomène est-il dû au partage traditionnel entre public (masculin) et privé (féminin), encore valable de nos jours (Oeser, 2007).

Les références à la religion ne sont pas beaucoup plus présentes en classe de seconde qu'en classe de sixième, mais les élèves de seconde utilisent bien plus souvent les termes « religion(s) " ou « religieux(se) ». Il y a autant de références à la Saint-Barthélemy en sixième qu'en seconde (la Réforme étant au programme en primaire comme au collège), mais les lycéens font davantage de nuance entre catholiques et protestants : la variable classe de seconde est en très forte corrélation avec les termes " protestant(s) » et « catholique(s) ». Il semble que la distinction soit plus difficile à mettre en mots pour les élèves de sixième. En ce qui concerne la Saint-Barthélemy, l'événement n'est jamais mentionné par les élèves

2.Voir à ce sujet la contribution précédente d’Églantine Wuillot (p. 83-99). 
de l'enseignement privé alors même que ces derniers utilisent 2 fois plus souvent les termes "religion(s) ", « religieux(se) », "chrétien » ou " christianisme ». On perçoit donc non seulement une forte insistance sur les religions de la part des élèves du privé, mais aussi une tendance à omettre certains aspects des religions, qui se confirmera au fil de l'analyse.

Au regard de la question qui nous occupe, le corpus corse est un peu différent du corpus continental. Si les élèves corses mobilisent les termes " religion ", " religieux(se) ", " chrétien " ou " christianisme " autant que les continentaux, ils parlent 2 fois plus souvent de « religions » au pluriel. La présence importante de la religion - notamment catholique - dans l'espace public corse conduit peut-être les élèves à y faire particulièrement référence tout en mobilisant une vision moderne et tolérante de celle-ci, qui insiste sur le pluralisme, d'où l'usage fréquent du pluriel. Ainsi : "Bastia, Propriano, etc. Ces villes sont aussi belles les unes que les autres. Car il y a beaucoup de religions et on se comprend. " [récit $\mathrm{n}^{\mathrm{o}} 3812$, collège] ; et encore : «La France est synonyme de religion et de mélange de religions. » [récit $n^{\circ} 4182$, lycée]

Pour éclairer ces récits qui mettent l'accent sur une forme de lien social ou de "vivre ensemble " fondé sur la compréhension mutuelle entre religions, on peut les mettre en perspective avec les déclarations de certains responsables scolaires. Par exemple, pour justifier sa décision de fermer les écoles durant la fête de la Nativité (localement : fête de la Santa di Niolu), déclaré jour vaqué en septembre 2014, le recteur de l'académie de Corse, Michel Barat, invoque un «lien social [qu'il] tien[t] à protéger [...], qui se désagrège ailleurs dans une démocratie qui individualise et non pas dans une démocratie qui réunit» (Barat, 2014). La différence avec le continent est soulignée : «ce qui manque sur le continent, c'est pas la qualité de l'enseignement [...], c'est pas la qualité de l'organisation ", mais précisément "le lien social» (ibid.). Si le recteur se limite à louer la corsitude ( "la corsitude, c’est aussi un lien qui fait réussir nos élèves »), quelques récits frappants, bien que minoritaires, vont plus loin en valorisant explicitement le christianisme : «La Corse est un pays de religion chrétienne ! [...] Dieu l'a créée.» [récit n 4493, collège]; de même : «La Corse est entièrement Chrétienne. » [récit nº 4494, collège] Cela dit, les récits corses qui insistent sur la ou sur les religions restent assez clairsemés. L'ensemble s'apparente davantage aux discours des élèves continentaux qu'à ceux des élèves réunionnais, pour lesquels le lien social religieux est vraiment central (voir infra, "Comparaison avec La Réunion », p. 112-114).

Enfin, la fréquence des différents termes renvoyant à la religion n'est pas du tout corrélée à la variable "langue parlée à la maison ». Le fait de parler une langue étrangère (pour des raisons d'immigration ou de spécificité régionale) n'a donc pas d'incidence sur les références à la religion dans les récits. L'école publique exerce ainsi une influence sur ce que disent les élèves : les récits inspirés des cours sont différents de ceux qui mobilisent des sources "moins scolaires » et les récits issus du public sont 
différents de ceux du privé ${ }^{3}$. Par ailleurs, si les élèves d'origine linguistique différente ne produisent pas de récits différents, une hétérogénéité frappante différencie les filles et les garçons, ces derniers ayant une vision plus institutionnelle, politique et guerrière de la religion.

\section{LE SENS DES RÉCITS MÉTROPOLITAINS}

\section{Laïcisation et valorisation du christianisme}

Pour préciser ces observations et en faire apparaître de nouvelles, nous avons procédé à une classification des 5086 récits en 11 catégories, croisées ensuite avec 6 variables : établissement public ou privé, sources scolaires ou non, académie Corse, milieu rural, langue d'origine non française, sexe. Les références très lapidaires à la religion ont été exclues. Par exemple : "Il y eut la préhistoire avec les dinosaures, puis vint ensuite homo sapiens. Il y eut ensuite l'antiquité, naissance de Jésus Christ, de nombreux baptêmes. La renaissance fit son apparition. " [récit n ${ }^{\circ} 2238$, lycée] Ce type de référence très brève, qui ne permet pas de savoir vraiment ce que pense l'élève au sujet de la religion, a toutefois été pris en compte dans l'analyse d'occurrences (voir supra).

Ici, au contraire, seuls les développements significatifs sont retenus. Ceux-ci se retrouvent dans 193 récits au total. C'est peu, mais cette faible proportion (3,8\% du corpus) est significative : elle indique que les élèves privilégient autre chose que la religion dans l'histoire de France - la religion étant elle-même appréhendée sous l'angle politique. La plupart des 193 récits pertinents peuvent être regroupés en deux catégories génériques, elles-mêmes divisées en plusieurs sous-catégories (tableau 2).

Notons d'abord une majorité de récits "laïcisants ». Leurs auteurs considèrent en effet plus ou moins explicitement la laïcisation comme un processus central de l'histoire. Cent trente-neuf textes répondent à cette description. On peut les classer de la façon suivante :

Récits anticléricaux. Par exemple : "Le Moyen Age arrive avec ses cathédrales, la prise du pouvoir par l'Église et ses croisades sanglantes qui ont retardé le progrès de l'Europe de dizaines d'années. " [récit n 4066, lycée] De même : "Après cela il y eut le moyen-âge ; je n'aime pas cette époque, c'est une sorte de rabâchage incessant de rois qui se succèdent,

3. Sur la difficulté de distinguer les sources scolaires des sources non-scolaires, voir la contribution deVincent Chambarlhac dans ce volume (p. 39-47). Pour notre part, nous avons retenu uniquement la première réponse des élèves à qui il était demandé de hiérarchiser entre diverses sources. Précisons que l'influence scolaire dont il est ici question peut se comprendre de différentes façons. On peut s'interroger : l'école est-elle à l'origine des représentations des élèves ou les sources scolaires permettent-elles simplement aux élèves d'organiser et d'exprimer des idées qui étaient déjà plus ou moins présentes dans leurs esprits ? Cette question vaut particulièrement pour la différence constatée entre public et privé, qui n'est sans doute pas due à la seule influence scolaire, les familles pouvant choisir le mode de scolarisation (public ou privé) selon telle ou telle conviction préalable. 
de clercs qui volent la population "pour dieu”." [récit n 3051, lycée] De manière générale, il s'agit de discours très critiques ("volent ", " sanglantes »), axés sur l'aspect institutionnel (" pouvoir », « clercs »).

Tableau 2. Répartition des récits "laïcisants " et "chrétiens".

\begin{tabular}{|l|c|}
\hline Total des récits & 193 \\
\hline Récits laïcisants (72\%) & 139 \\
\hline - anticléricaux & 13 \\
\hline - traitant la religion comme phénomène passé & 11 \\
\hline - mettant en équivalence religion et clergé & 9 \\
\hline - insistant sur l'intolérance politico-religieuse & 58 \\
\hline - parlant de la religion exclusivement au prisme du pouvoir & 20 \\
\hline - parlant de la religion exclusivement au prisme de la läicité & 17 \\
\hline - décrivant le baptême de Clovis comme un acte opportuniste & 11 \\
\hline ou un acte de pouvoir & 30 \\
\hline Récits chrétiens (16\%) & 20 \\
\hline - considérant avec bienveillance l'apport historique du christianisme & 6 \\
\hline - pro-chrétiens & 4 \\
\hline - soulignant l'apport religieux du baptême de Clovis & 24 \\
\hline Autres genres de récits (12\%) & \\
\hline
\end{tabular}

Récits décrivant la religion comme un phénomène fondamentalement passé. Ainsi : "Pour moi, l'histoire de France n'est pas quelque chose d'extraordinaire. Certes, il y a des moments importants bien connus comme les religions, Louis XIV... Ce que je trouve le plus important à dire, c'est le développement de la France depuis des dizaines d'années. " [récit $n^{\circ} 5700$, lycée] ; et encore : «De nombreuses guerres avec différents rois, différents règnes, 1 re guerre, 2è guerre mondiale, puis aussi les famines, la peste, les religions, les émigrations. Mais moi dans tout cela ce qui m'a le plus marqué c'est Hitler." [récit n 1904, lycée] Dans ces récits, "les religions " sont reléguées à une époque jugée moins "importante » que l'époque contemporaine ou associées à des événements qui n'ont plus cours en France (guerres, famines, peste).

Récits mettant plus ou moins en équivalence religion et clergé. Par exemple : "Puis il y a eu le Moyen-âge, il y a eu les chevaliers : la noblesse, puis les paysans, puis il y a eu les Chrétiens : le clergé." [récit n ${ }^{\circ} 5442$, collège] De même : "Puis au XVIII ${ }^{\mathrm{e}}$ siècle, nous sommes au siècle des lumières, les philosophes contestent le pouvoir de la religion, du régime en place.» [récit n ${ }^{\circ} 4226$, lycée] On voit bien ici la quasisynonymie entre chrétiens et clergé ainsi que l'association entre religion, pouvoir et régime. Cependant, ces discours ne sont pas explicitement anticléricaux. 
Récits insistant sur l'intolérance politico-religieuse. "Il y avait des rois qui étaient très sévères aussi pour la religion : ceux qui ne croyaient pas en Dieu, on leur coupait la tête ou on les brûlait. " [récit no 4252, collège] ; "Louis XIV, dit le roi Soleil, a détruit l'Édit de Nantes et voulut exterminer tous les Protestants. " [récit n ${ }^{\circ} 2708$, collège]

Récits parlant de la religion sous le prisme exclusif de la laïcité. Par exemple : "Aujourd'hui, en France, il n'y a pas de religion, d'opinions imposées. On fait "comme on veut", on peut croire en n'importe quel Dieu." [récit n ${ }^{\circ} 5913$, lycée] ; et encore : «La France est un pays laïque (c'est-àdire indépendant à l'égard des religions). " [récit n ${ }^{\circ}$ 5132, collège] Ici, le terme religion(s) est bien présent, mais on voit que le thème central est la laïcité.

Récits parlant de la religion sous le prisme exclusif du pouvoir. "Tout cela commence par la monarchie absolue, ce qui est un roi qui commande tout le monde. "Je suis le roi, je représente Dieu, personne ne doit me désobéir." " [récit n 4748 , lycée] Sans être anticlérical et sans établir explicitement une équivalence entre religion et clergé, ce type de discours mobilise un élément religieux (ici «Dieu») pour développer un thème strictement politique (au sens de la pratique du pouvoir).

Récits décrivant le baptême de Clovis comme un acte opportuniste ou un acte de pouvoir. "Je pense à Clovis, premier roi des francs, à son baptême en 496 où il devient allié de l'Église pour obtenir des aides financières." [récit n²205, lycée] ; "Les Francs ont finalement dominé (avec Clovis qui devient chrétien et qui impose sa religion au reste de la France).» [récit n 3933, lycée] Dans ces extraits, le baptême est jugé peu sincère, conduisant par ailleurs à une contrainte ("impose»).

À l'opposé, on relève 30 récits plus ou moins chrétiens, au sens où le christianisme y est perçu comme positif. Cette catégorisation peut être évidemment critiquée. Par exemple, l'extrait de récit suivant, qui considère avec bienveillance l'apport historique du christianisme, n'est pas forcément chrétien : "L'avancée du christianisme a provoqué le temps des cathédrales et de la grandeur. " [récit n ${ }^{\circ} 720$, lycée] Nous entendons cependant chrétien dans un sens large, confessionnel aussi bien que culturel. On peut alors procéder à un classement en trois catégories particulières :

Récits considérant avec bienveillance l'apport historique du christianisme. Par exemple : "La France est une vieille civilisation chrétienne avec beaucoup de dates et de faits importants et beaucoup de souverains. » [récit n 3552 , lycée] ; ou encore : "Les rois s'étant convertis à la religion chrétienne, apportèrent au pays cette volonté de prier ensemble le même dieu, autre élément de notre patrimoine historique. » [récit n ${ }^{\circ} 644$, lycée] Bien que ces récits ne soient pas chrétiens au sens strict, on ressent en les lisant une perspective culturelle-patrimoniale qui suggère que le christianisme fait partie de l'identité française.

Récits pro-chrétiens. Citons deux cas à cet effet : "La franc-maçonnerie continue à détruire la France qui jadis était un beau pays catholique.» 
[récit $n^{\circ} 3368$, lycée] ; «Les croisades donnent une vraie valeur au christianisme. » [récit no 2586, lycée]

Récits soulignant l'apport religieux du baptême de Clovis. Ainsi : "Clovis Ier qui offrit à la France la chrétienté. » [récit no 5022, lycée] L'événement est ici considéré comme sincère, la chrétienté de la France n'étant pas imposée, contrairement à l'exemple mentionné plus haut.

On trouve donc $72 \%$ de récits laïcisants et $16 \%$ de récits chrétiens ${ }^{4}$. L'histoire subjective, celle que racontent les élèves, correspond bien à ce que Marcel Gauchet appelle la "laïcisation objective ", qui selon lui a formé la conscience nationale. L'analyse fine des textes permet de montrer que l'école est un vecteur de cette vision laïcisante ; en tout cas, elle encourage l'expression d'une vision laïcisante préalable. Prenons en exemple les récits anticléricaux, ceux qui considèrent la religion comme un phénomène fondamentalement passé, ceux qui font une équivalence entre religion et clergé, et ceux qui décrivent la religion exclusivement « en négatif " au prisme du pouvoir ou de la laicité. Ces trois sous-catégories regroupent 68 récits au total, dont $85 \%$ sont inspirés de sources scolaires (cours ou manuels). Sachant que le corpus métropolitain compte 59\% de ce type de sources, il s'agit d'une proportion élevée. De même, $78 \%$ des 58 récits qui insistent sur l'intolérance politico-religieuse ont des sources scolaires. Par contraste, ces dernières n'inspirent que $57 \%$ des récits chrétiens, proportion proche de celle qui caractérise l'ensemble du corpus métropolitain. Si l'on précise que les récits chrétiens sont majoritairement produits par des élèves d'établissements privés, l'assertion suivante semble plausible : tout en étant racontée par les élèves dans un contexte relativement peu contraignant, l'histoire laïcisante est influencée par le curriculum de l'école publique. La laïcisation « objective » décrite par Gauchet serait donc également vivante sur le plan subjectif, dans les consciences des élèves, par le biais de l'histoire enseignée conçue plus généralement comme un outil de socialisation politique (Lantheaume, 2003).

\section{Illustration}

Des récits typiques permettent d'illustrer ces points. À bien des égards, dans le corpus métropolitain, l'Église est présentée comme dangereuse, intéressée par le profit et composée de gens malhonnêtes. Par exemple : "Quiconque contestait l'Église, contestait la monarchie était souvent

4. Il reste donc $12 \%$ de récits : ceux-ci ont été difficiles à classer dans une des deux catégories susmentionnées, le plus souvent parce qu'ils sont très lapidaires, peu clairs ou très descriptifs. Ils sont analysés ensuite. Précisons par ailleurs que les catégories utilisées ici se recoupent parfois : par exemple, nombre de récits anticléricaux insistent également sur l'intolérance politico-religieuse. Il a donc fallu, dans certains cas, « choisir » la catégorie la plus adaptée, ce qui pose un problème de méthode dans la mesure où le chercheur devrait s'abstenir, autant que possible, d'interpréter le discours des élèves. Nous avons bien sûr essayé de pratiquer cette abstinence, mais sans être certain d'y parvenir à chaque fois, tant la tâche est difficile - la sociologie d'inspiration ethnométhodologique l'a bien montré. 
condamné à mort. Des paysans ne pouvaient plus payer l'entrée dans l'église (surtaxée par les prêtres qui, forts de leur position, ne voulaient que s'enrichir).» [récit no 3772 , lycée] Avide de pouvoir, l'Église est aussi décrite comme intolérante : «L'Église prend peu à peu le pouvoir. Inquisition : le Christianisme persécute les autres religions. " [récit no 1926, lycée] Les agnostiques sont également visés, notamment lors « des guerres, celles où on pend les non-croyants. " [récit n 3638 , lycée] Cette époque cruelle n'est pas due uniquement aux clercs ou aux prêtres; elle est aussi associée à la naissance du Christ : "Puis, vint l'ère du Christ, cette ère où les guerres faisaient rage, où le sang giclait, tout cela à cause des religions. " [récit $\mathrm{n}^{\circ} 2852$, lycée] La présence de Jésus est même perceptible durant les croisades : "Moyen-âge avec les chevaliers et Jésus, il y a eu les croisades. " [récit $\mathrm{n}^{\circ} 5090$, collège] Dans ce contexte, il importe de contrôler les excès des religions, y compris le protestantisme, a priori moins violent, bien que « la religion protestante, [...] plus jeune que la religion catholique, [soit] sûrement en train de faire les mêmes erreurs [que la religion catholique] a faites quelques siècles plus tôt " [récit n ${ }^{\circ} 683$, lycée]. Ces jugements de valeur négatifs peuvent déboucher sur une conception très restrictive de la laïcité. Ainsi en est-il de cet élève corse, qui écrit : "Et puis tiens, parlons d'histoire : l'école est censée être LAIQUE, je trouve intolérable que certaines leçons traitent de RELIGIONS ! (Christianisme ou autre).» [récit nº 3997, lycée]

Par conséquent, dans les récits d'élèves, la mise à l'écart de la religion est constitutive de la France qui ne développe son identité qu'en opposition aux conceptions religieuses : "la France a pensé très "religieux" à l'époque du Moyen-âge, tout passait par Dieu. Ensuite elle a développé ses pensées avec le siècle des lumières. " [récit n ${ }^{\circ} 2822$, lycée] Cette mise à l'écart est tellement puissante qu'elle concerne parfois toute une époque et même au-delà. Si les nobles disparaissent, les paysans et les prêtres s'éclipsent aussi :

Les gens étaient croyants. Il y avait des paysans, des nobles et des prêtres. Les paysans avaient beaucoup de travail et les trois quarts étaient pour les nobles. Il y eut aussi la république donc un président et les citoyens pouvaient voter. Il n'y avait plus de nobles, de prêtres, ni de paysans. [récit $\mathrm{n}^{\circ} 4914$, collège]

Toutefois, la prépondérance de ce type de récits ne saurait masquer une minorité de récits qui contestent la vision républicaine de l'histoire. Par exemple, un élève scolarisé dans le public écrit :

Beaucoup d'éléments historiques sont allégés, car ils contredisent notre actuelle société. Je citerai par exemple les guerres de Vendée pendant la Révolution : le massacre des catholiques est apaisé aujourd'hui ; il y a aussi des avis péjoratifs de la royauté, dus à notre république actuelle. Les républicains pendant la révolution paraissent justes et bons. Pour moi ils étaient trop durs. [récit nº 2303, lycée] 
En somme, le corpus permet de montrer que les références à la laïcité et à la république, si fréquentes quand il s'agit d'évoquer la religion, ne sont pas seulement des formes rhétoriques mobilisées par les professionnels de la politique. Elles ne se réduisent pas à des idées imposées " d'en haut ", tant elles sont vivantes dans les récits produits " par le bas ${ }^{5}$ ".

\section{Autres caractéristiques des récits métropolitains}

Au vu de l'analyse précédente, on ne s'étonnera pas de constater la faible présence d'événements surnaturels dans les récits. L'histoire de France est sécularisée. Certes, Dieu ou Jésus intervient ici ou là, mais dans 11 récits seulement - une proportion insignifiante. Par exemple : "Jeanne d'Arc était une bergère qui promenait ses moutons. Un beau matin elle entendit la voie [sic] de Dieu. Dieu a dit à Jeanne de mener la guerre contre les Anglais. Jeanne dit oui à Dieu, elle partit voir Charles VII.» [récit $\mathrm{n}^{\mathrm{o}} 3512$, collège] ; de même : "L'histoire de France commence lorsque Jésus est né, Dieu a créé les hommes. Ils sont devenus des rois qui ont protégé leurs peuples et leurs nations. » [récit no 2345, lycée]

Le présent est également sécularisé : si le $\mathrm{xx}^{\mathrm{e}}$ siècle est souvent évoqué (guerres mondiales, Trente Glorieuses...), la religion en tant que telle n'y apparaît qu'à travers 13 récits $^{6}$ inspirés surtout de sources non-scolaires (9 sur 13). Comme on l'a indiqué précédemment, les élèves ont tendance à cantonner la religion au passé. Toutefois, cette tendance concerne moins les Corses et les ruraux, surreprésentés parmi les élèves faisant référence à la religion comme phénomène contemporain. L'hypothèse d'une influence religieuse diffuse, plus importante en Corse et en milieu rural, pourrait expliquer ce résultat. Enfin, notons que l'islam, lorsqu'il est évoqué au présent, ce qui est exceptionnel, est le plus souvent perçu au prisme de la laïcité ou du terrorisme (4 fois sur 7). Par exemple :

L'attentat des Musulmans qui voulaient faire s'écraser un avion sur Paris : un échec. [récit $\mathrm{n}^{\circ} 4174$, collège]

L'interdiction de porter le voile pour les femmes musulmanes qui vont travailler et surtout l'interdiction de porter la burka en lieu public en France $[\ldots]$ pour des raisons de sécurité car nul ne sait ce que la personne peut cacher en dessous de la burka.» [récit no 5997, lycée]

Cela découle logiquement de la vision politisée qu'ont les élèves de la religion, largement perçue comme violente et en conflit avec la modernité.

5. Certes dans un contexte scolaire, mais en dehors de toute évaluation scolaire et à la suite d'une question sollicitant des développements libres (voir l'introduction de l'ouvrage, p. 5-12).

6. Sans prendre en compte les confusions entre judaïsme et judéité (par exemple : « Hitler tentera de faire un génocide des personnes étant de religion juive » [récit nº 6037, lycée]), qui relèvent davantage d'une difficulté de compréhension que d'une véritable référence à la religion. 
En définitive, l'analyse permet d'étayer le constat récemment fait par Danièle Hervieu-Léger :

la « religion » est, dans notre culture nationale, associée au conflit et même à la violence. En un sens, le souvenir lointain de la Saint-Barthélemy fait partie de notre ADN national ! [...] Les débats sur la laïcité découlent directement de cette approche. (2013, p. 124.)

Cela pose évidemment la question difficile du rapport entre histoire et nation : les élèves ont-ils une conscience historique particulièrement aiguisée faisant obstacle à la " formation d'une nation » qui nécessiterait au contraire "l'oubli » (Renan, 1887, p. 284) ? Certes, beaucoup d'élèves ne semblent pas prêts à oublier les violences religieuses des siècles passés et ils se méfient aussi des prétentions politiques actuelles des religions. D'un autre côté, la présence finalement restreinte de la thématique religieuse dans le corpus global peut être elle-même considérée comme une forme d'oubli qui, laissant place à une conception résolument moderne et läque de la citoyenneté, faciliterait la formation d'une nation au sens de Renan. En tout cas, ce rapport spécifique à l'histoire, peu focalisé sur les religions en général et insistant sur leur aspect institutionnel, ne semble pas près de s'essouffler tant il est repérable dans les récits des jeunes collégiens et lycéens.

\section{COMPARAISON AVEC LA RÉUNION}

Comme on l'a noté en introduction, le corpus réunionnais (737 récits) est très différent du corpus métropolitain. Toute velléité de comparaison entre les deux corpus nécessite une analyse distincte du premier (voir le texte de Raoul Lucas, Mario Serviable et Stéphane Guesnet, p. 145163). Si les occurrences du terme "religion", au singulier, sont à peu près similaires, le mot "religions ", au pluriel donc, est employé 4,4 fois plus à La Réunion qu'en métropole, sans doute à cause de la pratique plurireligieuse de la majorité des Réunionnais et du culte des ancêtres qui s'exprime à travers un «schème rituel commun [qui] permet de jeter un pont d'un système religieux à l'autre et ouvre la voie à des parcours religieux multiples. " (Dumas-Champion, 2003, p. 87.) Cette hypothèse est corroborée par l'usage du terme "ancêtres ", généralement associé à l'idée de cultures, que l'on trouve 5 fois plus souvent à La Réunion qu'en métropole. Par exemple :

Nos cultures et nos ancêtres. [récit no 2133, collège]

Nos ancêtres té danse maloya, nou la continué amélior le maloya [...]. À La Réunion néna èn ta religions car nout ancêtres té vien de Madagascar, Afrique, Inde, Chine, Mayotte... [récit n 2137, collège]

La Réunion reste une île avec plein d'histoire. Il y a plein de cultures qui nous viennent des ancêtres. [récit nº 1167, lycée] 
Notons que le terme «cultures » est lui-même employé, sur l'île, dans une proportion 12 fois supérieure à son usage dans l'Hexagone.

Dans ce contexte, on ne sera pas surpris de découvrir, dans les récits réunionnais, une forte évocation de l'islam ${ }^{7}$. Bien que très minoritaire sur l'île, cette religion est en quelque sorte associée à l'identité culturelle «cumulative » de La Réunion. Par exemple :

L'île est bien connue pour son métissage, elle accueille une multitude de cultures (musulmans, Mahorais, Malgaches, Chinois, Créoles, Indiens) qui vivent ensemble dans la fraternité et la solidarité. [récit nº 3235, post-bac]

Notre île est réunie de plusieurs religions, cultures, donc de beaucoup de métissés qui viennent d'un peu partout du monde. Nous avons principalement les Malgaches, les Bouddhistes, les Musulmans, les Hindous. Au jour d'aujourd'hui la majorité de ce département est catholique. [récit n 1076, lycée]

Précisons que la proximité géographique entre les lieux de cultes est censée illustrer cet heureux assemblage : «Il y a beaucoup de religions mélangées et beaucoup de métissage [...]. Sur la ville de Saint-Louis, il y a une mosquée et à peine à 100 mètres se situe une église. " [récit $\mathrm{n}^{\circ} 1074$, lycée] L'islam est ainsi perçu comme l'élément d'un mélange religieux et culturel (ces deux aspects étant d'ailleurs peu distingués), tandis que les quelques élèves métropolitains qui font référence à l'islam l'évoquent plutôt dans sa dimension politique, notamment à travers le thème de la laïcité ou du terrorisme. Les occurrences du mot « laïcité » ou laïque sont d'ailleurs 3 fois moins fréquentes dans le corpus réunionnais que dans le corpus métropolitain.

À La Réunion, l'insistance sur les religions contraste fortement avec la référence à la religion dans les récits métropolitains. Les spécificités généalogiques de la modernité française expliquent sans doute cette différence. Alors que dans d'autres pays - notamment de tradition protestante -, l'État devait se séparer des confessions, l'État français ambitionnait de se séparer de la religion, en l'occurrence catholique (Gauchet, 1998, p. 51). Or, abolissant certaines spécificités culturelles (Guilhaumou \& Kaufmann, 2003), cette dynamique ne concerne pas la population réunionnaise qui, touchée par l'esclavage et construite sur la base de nombreuses migrations, reste globalement attachée à une multitude de rites religieux permettant à l'individu de retrouver des « racines » (Dumas-Champion, 2003). Outre son intérêt en soi, le corpus réunionnais fait ainsi ressortir, par contraste, des caractéristiques frappantes du corpus métropolitain. Il ouvre également à une conception tout à fait différente - et pourtant française - de la modernité, qui n'évacue pas les religions et ne les réduit pas à leur dimension politique. En témoignent les citations suivantes :

7. L'islam comme phénomène contemporain est proportionnellement 11 fois plus présent dans les récits réunionnais que dans les récits métropolitains. 
La Réunion est une île qui réunit plusieurs nations, religions. [récit $\mathrm{n}^{\circ} 2269$, collège]

De par son histoire et un brassage culturel, La Réunion est une terre de tolérance où cultures et religions diverses vivent en "harmonie". [récit $n^{\circ} 3125$, post-bac]

On a une multitude de religions, de couleurs, de races. [récit $\mathrm{n}^{\circ} 2120$, collège]

La Réunion est une île métissée où existent plusieurs religions, tous les citoyens ont une religion différente, comme la religion musulmane, chrétienne, hindoue et chinoise. [récit n ${ }^{\circ} 1081$, lycée]

À La Réunion il y a beaucoup d'ambiance lors des fêtes, anniversaires, baptêmes, communions... [récit no 2062, collège]

Loin d'être en conflit avec la modernité, les religions et leur métissage en sont ici constitutifs. Cette importante différence entre l'île et la métropole est d'ailleurs suggérée par plusieurs élèves. Par exemple :

Du fait de l'esclavage et de ses peuples d'origines différentes, la Réunion est un des départements les plus tolérants en ce qui concerne la multiculturalité. Les différentes religions se côtoient dans le respect de chacun. [récit n ${ }^{\circ} 3236$, post-bac]

Une autre enquête, qui adopte une perspective moins centrée sur la discipline historique, souligne :

[Les élèves] sont $82 \%$ à reconnaître que la religion a une place importante dans l'histoire et $59 \%$ à penser que parler de religion les aide à mieux comprendre ce qui se passe dans le monde. [...] Seule une minorité d'élèves a des jugements très négatifs sur les religions [...]. Les élèves prônent à leur manière une laïcité d'intelligence et de dialogue (Béraud \& Willaime, 2009, p. 241-242).

Si on les met en perspective avec la présente étude, ces résultats révèlent un double hiatus. Tout d'abord, entre les déclarations et la pratique : la défiance à l'égard des religions et l'omission de leur rôle dans l'histoire semblent plus fortes quand il s'agit de raconter l'histoire que quand il s'agit de déclarer leur importance. Ensuite, entre l'histoire en général et l'histoire de France. En effet, l'affirmation "la religion a une place importante dans l'histoire " (citation précédente) ne concerne pas spécifiquement la France, tandis que, comme on l'a vu, les élèves ne mentionnent guère la religion dans l'histoire nationale. S'ils le font, c'est dans une optique très politique et plutôt négative.

Le corpus analysé permet de contribuer à une réflexion plus globale sur ce qu'est la modernité française aujourd'hui, notamment dans son rapport à la religion. On sait que le républicanisme classique (Laborde, 
2010) est fortement remis en question par ceux qui considèrent la modernité elle-même comme une période plus ou moins révolue. Divers indices permettraient d'étayer l'idée d'un passage vers une ère " ultramoderne ", c'est-à-dire vers une nouvelle " conjoncture civilisationnelle " qui se manifeste par la "désabsolutisation de tous les idéaux séculiers qui, dans un rapport critique au religieux, s'étaient érigés en nouvelles certitudes. » (Willaime, 2007 a, p. 152, 155). Par exemple, le discours de Nicolas Sarkozy en 2007 au palais de Latran ${ }^{8}$ est parfois jugé comme étant "en phase avec l'ultra-modernité " (Willaime, 2009, p. 212). L'analyse des récits d'élèves permet d'aller plus loin dans le débat : les nouvelles générations expriment-elles une vision "ultramoderne » confirmant leur entrée dans un nouvel âge tout à fait différent ?

Les données à disposition infirment cette hypothèse. Les élèves se méfient de la religion, qu'ils décrivent majoritairement comme un phénomène institutionnel, un instrument de domination, levier du pouvoir, appréhendé au prisme de la laïcisation et appartenant surtout au passé. Sous ce rapport, pour sauver l'hypothèse de l'ultra-modernité, il faudrait supposer que les représentations étudiées ici sont en décalage avec les dynamismes contemporains, mais ce serait s'engager sur une pente normative que nous refusons d'emprunter. De même, faut-il remettre en cause l'enseignement scolaire actuel, qui influe significativement sur les récits laïcisants ${ }^{9}$, en promouvant par exemple un enseignement des faits religieux destiné à " reconnaître la profondeur symbolique du religieux ", à "prendre en compte la béance herméneutique [...] qui est au cœur des expressions religieuses ", à " explorer les sentiments religieux ", les faits religieux étant "immergés dans les sociétés " (Willaime, 2007 b, p. 45, $56,67)$ ? De tels objectifs font débat (Urbanski, 2015) et leur réalisation éventuelle ne sera pas facile, tant les élèves métropolitains sont influencés par une vision laïcisante, typiquement française, de l'histoire.

\section{Bibliographie}

BARAT Michel (2014), Intervention sur la radio corse Alta-Frequenza, 3 septembre, en ligne :www.alta-frequenza.corsica/1_info/1_actu/les_ eleves_de_corse_n_auront_pas_classe_ce_lundi_8_septembre_70246 (août 2016).

Baubérot Jean (2008), La Laïcité expliquée à M. Sarkozy... et à ceux qui écrivent ses discours, Paris, Albin Michel.

BArgel Lucie (2013), "Socialisation politique », dans Catherine Achin \& Laure Bereni (dir.), Dictionnaire genre \& science politique : concepts, objets, problèmes, Presses de Sciences Po, p. 468-480.

8. On sait à quel point la formule utilisée par l'ancien président de la République (" dans la transmission des valeurs et dans l'apprentissage de la différence entre le bien et le mal, l'instituteur ne pourra jamais remplacer le pasteur ou le curé ") irrita beaucoup de monde (Baubérot, 2008).

9. Cette influence pouvant se comprendre de différentes manières (voir supra, note 3). 
Béraud Céline \& Willaime Jean-Paul (coord.) (2009), Les Jeunes, l'école et la religion, Paris, Bayard.

DubET François (2012), "Éduquer dans un monde pluriel : François Dubet (entretien avec Geneviève Zoïa) », Tréma, no 37, p. 6-21.

Dumas-Champion Françoise (2003), "Pratiques religieuses plurielles à l'île de La Réunion ", dans Abel Kouvouama \& Dominique CochartCoste (dir.), Modernités transversales : citoyenneté, politique et religion, Paris, Éditions Paari, p. 71-87.

Gauchet Marcel (1998), La Religion dans la démocratie : parcours de la laïcité, Paris, Gallimard.

Hervieu-LÉger Danièle (2013), “"Religion”, “secte”, "superstition” : des mots piégés? (entretien)", Histoire, monde et cultures religieuses, n ${ }^{\circ} 26$, p. 121-127.

- (2003), "Pour une sociologie des "modernités religieuses multiples" : une autre approche de la "religion invisible" des sociétés européennes ", Social Compass, nº 50 (3), septembre, p. 287-295.

Guilhaumou Jacques \& Kaufmann Laurence (dir.) (2003), L'Invention de la société : nominalisme politique et science sociale au XVIII siècle, Paris, Éditions de l'EHESS, p. 123-161.

KaUfMANn Laurence (2010), «Faire collectif : de la constitution à la maintenance ", dans Laurence Kaufmann \& Danny Trom (dir.), Qu'est-ce qu'un collectif ? Du commun à la politique, Paris, Éditions de l'EHESS, p. 331-372.

Kintzler Catherine (2013), Penser la laïcité, Paris, Minerve.

LABORDE Cécile (2010), Français, encore un effort pour être républicains !, Paris, Éditions du Seuil.

Lantheaume Françoise (2003), "Solidité et instabilité du curriculum d'histoire en France : accumulation de ressources et allongement des réseaux ", Éducation et Sociétés, vol. 12, n 2, p. 125-142.

Oeser Alexandra (2007), "Genre et enseignement de l'histoire : étude de cas d'un Gymasium de la ville de Hambourg ", Sociétés E Représentations, $\mathrm{n}^{\circ} 24$, p. 111-128.

Renan Ernest (1887), Discours et conférences, Paris, Calmann-Lévy.

URBANSKI Sébastien (2015), "L'enseignement du fait religieux : une enquête dans trois collèges ", Recherches en éducation, no 21, p. 202-212.

Willaime Jean-Paul (2009), "Pour une sociologie transnationale de la laïcité dans l'ultramodernité contemporaine ", Archives de sciences sociales des religions, $\mathrm{n}^{\circ} 146$, p. 201-218.

- (2007 a), "Reconfigurations ultramodernes ", Esprit, n ${ }^{\circ}$ 3, mars-avril, p. 146-155.

- (2007 b), "Qu'est-ce qu'un fait religieux ? ", dans Dominique Borne \& Jean-Paul Willaime (dir.), Enseigner les faits religieux, quels enjeux ?, Régis Debray (préf.), Paris, Armand Colin, p. 37-91. 